\title{
A parenting programme to prevent abuse of adolescents in South Africa: study protocol for a randomised controlled trial
}

Lucie Cluver $^{1,2^{*}}$ D, Franziska Meinck', Yulia Shenderovich ${ }^{3}$, Catherine L. Ward ${ }^{4}$, Rocio Herrero Romero ${ }^{1}$, Alice Redfern ${ }^{1}$, Carl Lombard ${ }^{5}$, Jenny Doubt ${ }^{1}$, Janina Steinert ${ }^{1}$, Ricardo Catanho ${ }^{1}$, Camille Wittesaele', Sachin De Stone ${ }^{1}$, Nasteha Salah', Phelisa Mpimpilashe ${ }^{1}$, Jamie Lachman ${ }^{1,6}$, Heidi Loening ${ }^{7}$, Frances Gardner ${ }^{1}$, Daphnee Blanc ${ }^{1}$, Mzuvekile Nocuza ${ }^{1}$ and Meryn Lechowicz ${ }^{1}$

\begin{abstract}
Background: An estimated one billion children experience child abuse each year, with the highest rates in lowand middle-income countries. The Sinovuyo Teen programme is part of Parenting for Lifelong Health, a WHO/ UNICEF initiative to develop and test violence-prevention programmes for implementation in low-resource contexts. The objectives of this parenting support programme are to prevent the abuse of adolescents, improve parenting and reduce adolescent behavioural problems. This trial aims to evaluate the effectiveness of Sinovuyo Teen compared to an attention-control group of a water hygiene programme.

Methods/Design: This is a pragmatic cluster randomised controlled trial, with stratified randomisation of 37 settlements (rural and peri-urban) with 40 study clusters in the Eastern Cape of South Africa. Settlements receive either a 14-session parenting support programme or a 1-day water hygiene programme. The primary outcomes are child abuse and parenting practices, and secondary outcomes include adolescent behavioural problems, mental health and social support. Concurrent process evaluation and qualitative research are conducted. Outcomes are reported by both primary caregivers and adolescents. Brief follow-up measures are collected immediately after the intervention, and full follow-up measures collected at 3-8 months post-intervention. A 15-24-month follow-up is planned, but this will depend on the financial and practical feasibility given delays related to high levels of ongoing civil and political violence in the research sites.
\end{abstract}

Discussion: This is the first known trial of a parenting programme to prevent abuse of adolescents in a low- or middle-income country. The study will also examine potential mediating pathways and moderating factors.

Trial registration: Pan-African Clinical Trials Registry PACTR201507001119966. Registered on 27 April 2015. It can be found by searching for the key word 'Sinovuyo' on their website or via the following link: http://www.pactr. org/ATMWeb/appmanager/atm/atmregistry?_nfpb=true\&_windowLabel=BasicSearchUpdateController_ 1\&BasicSearchUpdateController_1_actionOverride=\%2Fpageflows\%2Ftrial\%2FbasicSearchUpdate\%2FviewTrail\& BasicSearchUpdateController_1id=1119

Keywords: Child abuse, Parenting, Low- and middle-income countries

\footnotetext{
* Correspondence: lucie.cluver@spi.ox.ac.uk

${ }^{1}$ Centre for Evidence-Based Intervention, Department of Social Policy \& Intervention, University of Oxford, Barnett House, 32 Wellington Square, Oxford OX1 2ER, UK

${ }^{2}$ Department of Psychiatry and Mental Health, University of Cape Town, Cape

Town, South Africa

Full list of author information is available at the end of the article
} 


\section{Background}

Worldwide, an estimated one billion children experience abuse each year [1], with the highest rates in low- and middle-income countries (LMICs) and in particular the World Health Organization (WHO) Africa region [2]. Although prevalence data are limited, new studies suggest increases in abuse during adolescence [3], which is also a time of important social, emotional and continued neural development [4].

Despite this, systematic reviews find that the vast majority of research into child abuse prevention is in highincome countries and with younger children [5]. In LMICs, three trials have tested abuse prevention parenting programmes, two targeted at children under 10 years old in South Africa [6] and Liberia [7] and one for 13year-olds and under in Burundi [8]. To date, there are no known randomised trials of a parenting programme to prevent abuse of adolescents in any LMIC [9].

Existing evidence - albeit from different contexts and child age groups - demonstrates good effect sizes of group-based parenting programmes that are grounded in social learning theory, problem-solving and acquisition of behaviour management skills [10]. Indeed, a recent systematic review showed high transportability of parenting programmes to address problem behaviour amongst younger children across high-income countries and contexts [11].

However, three major limitations exist to transportability to an LMIC. Firstly, many existing evidence-based programmes charge fees for training and manuals, making costs prohibitive for low-resource agencies and governments [12]. Other programmes require qualified health professionals for implementation, who are not available in the highest-need areas. Finally, many have technological components (e.g. videos and web-based modalities) that are as yet inaccessible in areas with poor electricity and internet access.

In response, an international collaboration was started in 2012, to develop and rigorously test a suite of child abuse prevention programmes for different age groups. Parenting for Lifelong Health (PLH) includes WHO, the United Nations Children's Emergency Fund (UNICEF) and academics from the global South and North, with donor partners, LMIC governments and the President's Emergency Plan for AIDS Relief (PEPFAR)-US Agency for International Development (USAID). PLH programmes are developed with participatory input from families in LMICs, for implementation by lay community workers, and have minimal equipment requirements. If shown effective in randomised trials, programmes will be freely available under licensing that prohibits any commercial or profit interests.

The adolescent programme, called Sinovuyo ('we have joy') Teen, has undergone incubation development and testing in very low-income rural and peri-urban areas of South Africa. Initial development used systematic reviews to identify effective components [10], input provided voluntarily from over 50 international academics and practitioners, and in-depth qualitative work with adolescents and caregivers [6]. Draft programme manuals were written in partnership between a local nongovernmental organisation (NGO; Clowns Without Borders South Africa) and academics from the universities of Oxford and Cape Town.

A first pilot pre-post non-controlled test with 30 caregiver-adolescent dyads showed initial reductions in abuse and adolescent behavioural problems, and no evidence of harm [13]. Concurrent qualitative research identified participant requests to incorporate economic strengthening approaches into the programme, to lessen family conflict over money. After adaptation, a second pre-post noncontrolled test with 115 dyads showed reductions in abuse, behavioural problems, depression and caregiver (but not adolescent) substance abuse, and improvements in positive/involved parenting and social support [14]. This second stage also piloted family budgeting and savings sessions, which were subsequently incorporated into the third version of the manual. The studies also found unanticipated high levels of programme dissemination within communities, particularly through church groups and community meetings.

At all stages, the project has been a close partnership between NGOs, the South African Department of Social Development and UNICEF. These agencies intend to decide whether to implement the programme within a provincial and national rollout, based on the results of this trial. Furthermore, an additional 17 countries in SubSaharan Africa, Central Asia, Eastern Europe and the Middle East have expressed strong interest in implementing the programme. The timing of this trial is thus critical for policy-making for child abuse prevention in LMICs.

Due to extenuating circumstances (high levels of civil and political unrest in the research sites in the first 6 months of the trial), this protocol is submitted after recruitment of the participants, and, therefore, falls outside the journal's usual policies. Data collection is still underway at the time of submission.

\section{Methods/design}

In this pragmatic cluster randomised trial in real-world settings, 40 rural and peri-urban settlements, containing 600 caregiver-adolescent dyads, are randomised to two parallel arms.

\section{Study Aims}

The study aims to compare the effectiveness of a 14session caregiver and adolescent programme with an attention-control group, amongst high-risk adolescents 


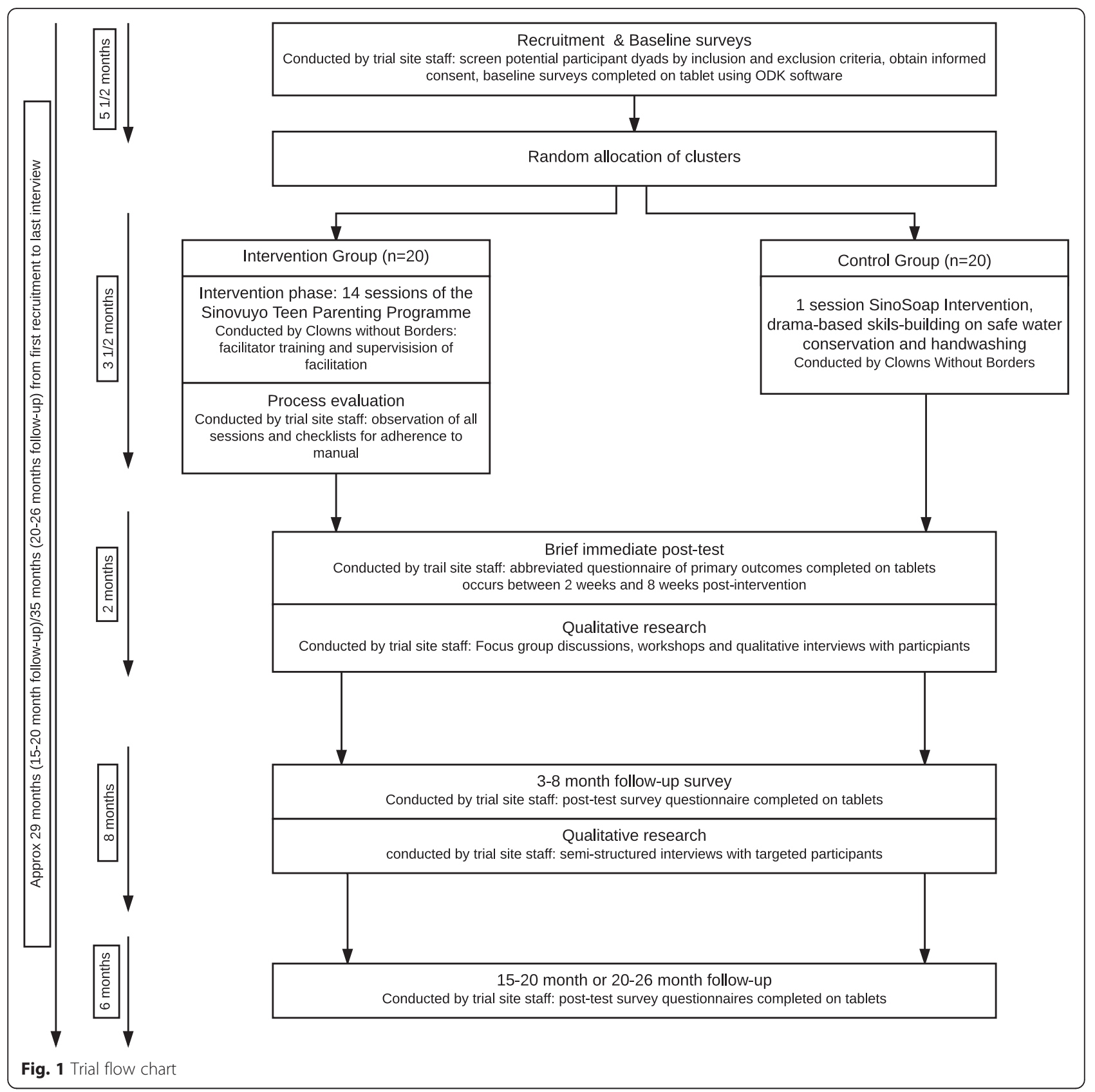

aged 10-18 and their families. Primary outcomes for the trial are (1) harsh and abusive discipline and (2) parenting. Secondary outcomes include adolescent externalising behaviour, parenting stress, mental health and social support. Exploratory outcomes include family financial coping, avoiding risk in the community, sexual harassment/abuse and educational engagement.

\section{Inclusion criteria}

\section{Communities}

The communities are rural and peri-urban settlements within a 1-hour driving distance of King William's Town, in the Eastern Cape province of South Africa. All areas have high rates of unemployment, poor infrastructure and high HIV/AIDS prevalence [15].

\section{Participants}

Adolescents are between 10 and 18, and either sleep in the same dwelling for at least four nights a week as their primary caregiver or have regular contact with them. Adolescents and their primary caregivers gave informed consent to participate. Recruitment followed pragmatic trial principles of closely approximating methods of inclusion in NGO or government services. Families were 
referred by a range of social services, schools and local chieftains, and were also able to self-refer as struggling with an adolescent. All families completed a brief screening questionnaire asking if there were regular arguments in the home.

\section{Exclusion criteria}

Following pragmatic trial principles, there were no exclusion criteria for families. There were no requirements for a biological relationship between caregiver and adolescent. Communities required approval from local traditional or political leaders (chieftains and ward councillors), and were estimated to be safe enough (during daylight hours and with local support) to hold a parenting group without serious risk to the participants. If a participant had such severe learning disabilities that they were unable to consent to participation, they were not included in the study for ethical reasons.

\section{Control sites}

Control rural and peri-urban settlements receive a onesession hygiene programme called SinoSoap. This is implemented by the NGO Clowns Without Borders South Africa and involves drama-based skills-building on safe water conservation and handwashing for children. All participating families in control sites receive a 'hope soap': a bar of soap containing a small toy that is only accessible when the soap is used.

\section{Intervention sites}

Intervention rural and peri-urban settlements receive the 14-session parenting programme called Sinovuyo Teen. Weekly sessions take place in the communities (church and community halls, schools and under trees).

\section{Programme and training}

The intervention programme is implemented by locally recruited unqualified community members and local social auxiliary workers. All programme facilitators received 1 week's initial training and weekly supervisions from Clowns Without Borders South Africa. The training was participatory and activity-based, and training materials are being developed for free availability.

The programme is based on evidence-informed parenting principles, such as praising each other, managing anger and stress, joint problem-solving, non-violent discipline, rules and routines, keeping adolescents safe in the community, and responding to crises (see Table 1). It uses collaborative problem-solving techniques (not didactic methods) and traditional stories, role-play, modelling and stress reduction activities [16-18].

Ten programme sessions are joint with caregivers and adolescents, and four sessions have separate components to allow sensitive discussions. Participants are encouraged
Table 1 Overview of intervention session topics

\begin{tabular}{lll}
\hline Session & Content & Mode \\
\hline 1 & $\begin{array}{l}\text { Introducing the programme and defining } \\
\text { participant goals }\end{array}$ & Joint \\
2 & $\begin{array}{l}\text { Building a positive relationship through } \\
\text { spending time together }\end{array}$ & Joint \\
3 & Praising each other & Joint \\
4 & Talking about emotions & Separate \\
5 & What do we do when we're angry? & Separate \\
6 & Problem-solving: putting out the fire & Joint \\
7 & Motivation to save and making a budget with & Joint \\
8 & Our money & Separate \\
9 & Dealing with problems without conflict I & Separate \\
10 & Establishing rules and routines & Joint \\
11 & Ways to save money and making a family saving plan & Joint \\
12 & Keeping safe in the community & Joint \\
13 & Responding to crisis & Joint \\
\hline
\end{tabular}

to engage in home practice in the week following each session. For participants unable to attend sessions due to illness, disability etc., khaya (home) catch-ups are arranged to give brief session content at home or in the hospital. A simple lunch is included at the beginning of each session as many participants find concentration difficult due to hunger.

\section{Randomisation}

Stratified randomisation was used. There were 40 eligible study clusters, 32 rural and eight peri-urban clusters, representing the two strata. Complete randomisation was done for clusters within strata in a 1:1 ratio for the intervention and control arms. Following the Cochrane guidelines and to reduce the possibility of recruitment bias [19], randomisation was performed by the study statistician (CL) after recruitment of clusters and before the intervention started, using a random number generator in Excel.

\section{Allocation concealment}

Blinding of patients and programme implementers is not possible because participants know whether they are receiving a parenting or hygiene programme. However, the trial statistician carrying out randomisation and analyses is blinded, and all efforts are made to keep data collection research staff blinded as to allocation for as long as possible. Due to the nature of the intervention (i.e. families displaying home practice sheets or certificates on their walls, and children in villages singing programme songs from either Sinovuyo Teen or SinoSoap), blinding is not always maintainable. To alleviate this, audio computerassisted self-interview (CASI) methods are used, and 
training of data collectors included consistent administration and awareness of biases.

\section{Measurement points and methods}

Primary caregivers and adolescents complete measures independently and in private using a tablet with data collector support at pre-test and 3-8 months post-intervention. Tablet-based questionnaires were designed to be engaging, with embedded activities and pictures, and modified scale responses using colours etc., for participants with low literacy. All questionnaires and audio-CASI sections were prepiloted with local adolescents and caregivers. Open-source software was used, and all questionnaires will be made freely available for other researchers.

Due to the high mobility of adolescents at the beginning of the new school year in January and the national government plans to scale the programme nationally in 2016, an additional brief immediate post-test data collection point was added, with a subset of outcome measures, to assess safety for this unanticipated scale-up. The 3-8-month post-test has been extended due to substantial levels of civil and election-related violence in the study areas, which have necessitated closing down the fieldwork for around $50 \%$ of the time. A 15-20-month or 20-26-month post-test is planned but will depend on financial resources, especially given the unanticipated costs related to violent community protests.

To increase participant retention in the study, we will continue to hold community meetings, and to work with chieftains and local councillors in all sites prior to returning for data collection. Only data on reasons for non-participation will be collected from participants who choose not to continue in the study.

\section{Measures}

All measures were translated into Xhosa and backtranslated. Interviews take place in homes and community settings, using tablet-based questionnaires with audioassisted interviews for stigmatised measures (child abuse, HIV/AIDS etc.). The research team are recruited locally but do not conduct data collection in their home areas, and are trained for a month on research ethics and in working with vulnerable children and families. All questionnaires are available at www.youngcarers.org.za.

\section{Primary outcomes}

Abusive parenting (physical abuse, emotional abuse and neglect) is measured using an adapted version of the International Society for Prevention of Child Abuse and Neglect Child Abuse Screening Tool (ICAST-Child, 18 items, and ICAST-Parent, 22 items) [20, 21] for use in intervention studies. Positive and involved parenting (16 items), monitoring and inconsistent discipline (16 items) and corporal punishment (five items) are measured using the Alabama Parenting Questionnaire (parent and child versions) [22].

\section{Secondary outcomes}

Adolescent behaviour problems uses the Child Behavior Checklist [23] rule-breaking and aggressive behaviour subscales (35 items). Parenting stress is measured using the Parental Stress Scale [24] (18 items). Caregiver depression (caregiver report only) is measured using the Centre for Epidemiologic Studies Depression Scale (20 items) [25]. Adolescent depression (adolescent report only) uses the short-form Child Depression Inventory (ten items) [26]. Adolescent suicidality is measured with the Mini International Neuropsychiatric Interview for Children and Adolescents [27]. Social support (for caregivers and adolescents) is measured using the Medical Outcomes Study Social Support Survey [28].

\section{Exploratory outcomes}

Family financial coping uses items assessing shortages in monthly budgets for purchasing meat, electricity etc., and the level of emotional stress experienced as a result, plus items on capacity to respond to emergencies, and borrowing and savings behaviours [29-31]. Planning for avoiding risk in the community is measured using the adapted Parent Teen Sexual Risk Communication Scale III (four items) [32], and five items from the Parent Communication Scale [33]. Exposure is measured using items from the National Survey of HIV and Sexual Behaviour amongst Young South Africans [12, 13], sexual abuse items from the ICAST, and exposure to community violence using three items based on risks identified in the Victimisation/Witnessing of Community Violence subscales of the Social and Health Assessment (SAHA) $[34,35]$. Education is measured using four adapted items of the SAHA academic motivation scale [36], with school attendance, grade repetition and school records of the South African standardised Annual National Assessment Learner Report if this is conducted as intended in 2016 [37]. Attitudes towards gender norms in relationships uses four items from the Gender Equitable Men scale [38].

\section{Process evaluation assessments}

Process evaluation assessments include implementer records of home visits, programme attendance rates, as well as independent observations of participant engagement and implementer fidelity and adherence during sessions. In addition, focus groups with implementers, participants and data collectors explored these topics qualitatively. In the pre-test questionnaire, participants self-assessed their motivation to improve their relationship with their adolescent/ caregiver, intentions to participate in both intervention and 
control arm programmes, and estimated difficulties and utility of attendance.

\section{Qualitative assessment}

The collection of linked qualitative data aims to understand how policy, service delivery, and social and economic factors may impact the effectiveness and scalability of the intervention. This focuses on: (a) recommendations of local staff delivering the programme, (b) family experiences of the parenting programme in the wider context of their lives and (c) policy and programming-level considerations for scaling a parenting programme in South Africa. The qualitative research study is conducted in partnership with UNICEF's Office of Research - Innocenti. Ongoing qualitative methods throughout the pilot and randomised trial stages (in English and Xhosa) include record analysis; semi-structured interviews with local and international NGOs, government partners and implementing partners; elite interviews with South African policymakers at the local, provincial and national levels; focus group and individual interviews with beneficiaries and implementers, including interactive activities and participatory visual methodologies; and programme workshop observation.

\section{Mediating and moderating pathways}

Potential mediating pathways that have been shown to mediate change in primary outcomes in other studies of child abuse prevention, will be explored. Given that no known randomised controlled trials (RCTs) have tested a parenting programme for families with adolescents in a LMIC, these mediation analyses will be tentative and based on the programme's theory of change, thus including potential mediators of change in parenting and child abuse such as parenting stress, parent mental health and social support. However, based on more substantial literature on parenting as a mediator of change in youth outcome, if there are main effects on the secondary outcomes of adolescent behavioural problems and mental health, we plan to examine changes in positive and harsh parenting as mediators of change in adolescent outcomes.

Potential moderating factors of programme effects will also be examined. Again, given the novel nature of this study, these are tentatively hypothesised. Potential moderators (see below) will include family AIDS-illness/ death, measured using verbal autopsy/illness questionnaires validated for high-prevalence areas [39]; poverty measured using the National Food Consumption Survey [40] and the South African Social Attitudes Survey [41]; and caregiver experience of maltreatment as a child and current gender-based violence measured using the ICAST-Retrospective (15 items) [42], five items from the revised Conflict Tactics Scale [43] and five items from the 15-item sexual relationship power scale [44]. Potential moderators will also include access to social protection provisions such as grants, community gardens and soup kitchens. We prefer not to predict whether poverty, family psychosocial or illness-related factors will increase or reduce programme effects, since the parenting intervention literature is very mixed on the direction of these moderator effects $[45,46]$. We will also examine variations in programme effects based on attendance and participation in the intervention, and implementer fidelity.

Potential mediation and moderation effects will be tested using structural models (either using Hayes' PROCESS path models or structural equation modelling depending on variable type [47]), and ideally using three data points at baseline, 3-8 months post-intervention and 15-20 and 20-26 months post-intervention. If the final follow-up is not possible due to financial or practical constraints, the models will use change scores of the mediating/moderating variable from pre- to post-intervention and then analyses should clearly be interpreted with caution.

\section{Socio-demographic covariates}

Socio-demographic covariates include age, gender, disability level, urban/rural location, household structure, caregiver-child relationship, household employment and HIV/AIDS-related stigma.

\section{Statistical analysis plan}

The sample size calculation was conducted by YS using the ICAST child abuse measure as the basis as it is the main outcome of policy interest. We estimated the range of intra-cluster correlation coefficients up to 0.08 based on pilot testing in the study area. We note that there are a large number of zero values in the reporting of child abuse in any pragmatic sample. Using Optimal Design software [48], 40 clusters with 12 families per cluster were required for a minimum detectable effect size of 0.36 for a desired power of .80 with a significance level of $5 \%$ with a two-tailed test. To account for attrition of up to $20 \%$ within each cluster, the target sample size was set at 600 families (40 clusters with 15 families per cluster). Our estimate of expected effect size was based on the effect sizes for maltreatment and parenting outcomes in a recent review of parenting programmes for child maltreatment prevention [49]. This showed an average programme effect of 0.2 , but the present study was limited by financial constraints, so will need to reach an effect of 0.36 to find significance.

\section{Types of analysis}

A baseline table reflecting the demographic profile of the households, caregivers and adolescents by arm will be compiled. Statistical analyses will be by intention to treat, with an additional per-protocol analysis. For the analysis of the primary outcomes, the suitability of using linear regression models will be checked. For primary 
outcomes that can be analysed with this approach, a hierarchical linear mixed effects model will be used to evaluate the intervention effect with the cluster and participant (caregiver or adolescent) as the nested random effects. To account for dropouts in the intention-to-treat analysis, the baseline measurement will be part of the repeated outcomes and estimation of the intervention effects will be via maximum likelihood. The significance of the intervention effect will be based on the significance of the arm by the time interaction effect. The models will include the stratification as a fixed effect. The impact of the missing data on the estimated intervention effect will be checked by imputing missing outcome data (ten imputations) using complete baseline and follow-up data and running the same models. For a primary outcome that does not meet the criteria for an individuallevel analysis, an analysis at the cluster level will be used using a standard linear regression model or a nonparametric quantile regression model. These models will allow for the comparison of arms, adjusted for the stratification and baseline cluster value. A similar sensitivity analysis will be done for the cluster-level analysis based on the imputed data. The primary and secondary outcomes will be analysed separately for caregivers and adolescents and a joint outcome model will be done as an exploratory analysis to understand the dependence of outcomes within a dyad. The impact of adherence to the intervention (number of sessions attended) will be assessed using the same models but restricted to the intervention arm.

\section{Stopping procedures}

An independent trial steering committee has been established. Two pilot studies showed no evidence of harm [13]. If there are any indications of negative effects, as noted in process observations or participant reports, the principal investigator and partner NGOs will be alerted, and preventative action taken.

\section{Modifications to the protocol}

Any changes to the protocol will be communicated to the relevant partners and funding bodies via email. The current protocol at the Pan-African Clinical Trials Registry will be updated online.

\section{Coordinating centre}

The coordinating centre at the University of Oxford consists of research and academic staff. It provides administrative and scientific support and runs the fieldwork component of the research. Oxford and the South African site jointly run safety responses to political violence, through WhatsApp messaging for instant communication of unsafe areas and evacuation of staff when needed.

\section{Steering committee}

The steering committee is composed of an independent, international group of academics with experience in running randomised trials of parenting interventions in LMICs, and child protection and child abuse prevention specialists. It provides scientific support and data monitoring where needed. The conduct of the trial is reviewed with this committee every 6 months.

\section{Data management}

Only a small number of the research team have access to personal identifiers, which are used to match caregiver and child interviews. Using a password-protected internet network, tablet devices transmit participant responses to a password-protected server where the data are automatically captured. Questionnaires sent to the server cannot be altered. Data collected on tablets cannot be accessed unless a key is entered, which is required when they are uploaded to a central network server, which is hosted by the University of Oxford. Thus, the data are protected from both local-server failure and confidentiality breaches. Non-electronic data are stored in a locked filing cabinet. In reporting the findings of this study, names will be omitted and only general locations in which the study took place will be reported. Data will be made available at Data Archive UK or a similar open-access archive. Non-anonymised data will be kept for up to 5 years in locked cabinets.

\section{Capacity-building and resource-sharing}

The trial aims to build capacity in low-resource contexts. This includes recruitment and intensive training of unqualified local staff, with provision of financial support for educational needs and recruitment and training of local community members in programme implementation. All study materials (qualitative and quantitative) will be freely available via the UNICEF and WHO websites. Doctoral and pre-doctoral students from LMICs are included in the study team.

\section{Dissemination Community-level}

The findings will be disseminated to all local communities, local leaders, NGOs and government departments through presentations, and a core research team will remain in the field site for 6 months to do this. At the policy and academic levels, ongoing dissemination is being carried out at academic and professional conferences and to international NGOs, LMIC governments and policymakers with regards to the development and testing of the intervention. 


\section{Discussion}

This study has potentially substantive scientific, policy and programming value. It is the first known randomised trial of a programme to prevent abuse of adolescents in a LMIC. It uses a pragmatic cluster RCT design, to provide maximum relevance to programming in low-resource settings. It is located in an area and with a population experiencing multiple concurrent challenges - and as a result of this, a number of key practical issues have emerged in beginning this trial. The study site has experienced increasing civil unrest, including violent riots related to service access, political rallies, xenophobic violence and protests against corruption. These have caused staff safety concerns, and disruptions to both data collection and intervention programming. Extended periods without electricity, drought and flooding have caused operational delays. Violent crime (hijacking of project vehicles etc.) has been rare but has required restructuring of research processes to increase staff security. Violent armed conflict between private taxi operators and petrol-bombing of roads have restricted transport of staff to field sites. Despite these challenges, the value of a pragmatic trial design is clear: increased external validity to assist decisions by policymakers. The strong interest shown by multiple countries and agencies in this programme highlights the perceived need for effective interventions to combat abuse amongst adolescents, and the importance of rigorous research evidence in this area.

\section{Trial status}

At the time of the revised submission of this manuscript in June 2016, the trial is ongoing. Recruitment commenced in March 2015 and a total of 1108 participants (554 caregiver-adolescent dyads) have been recruited and completed baseline assessments. Despite some delays due to violent protests in programme areas, the intervention took place with all 14 sessions conducted in 19 village/peri-urban clusters and 13 sessions conducted in one cluster. Altogether, $87 \%$ of caregivers and $85 \%$ of adolescents received $>90 \%$ of the sessions through groups or home visits. A brief immediate post-test data collection with a subset of primary outcomes was completed in December 2015, showing a $91 \%$ caregiver and $92 \%$ adolescent retention rate. These immediate results were primarily to determine safety and initial effectiveness for policymakers planning the South African national scale-up. The 3- to 8-month follow-up data collection was due to complete in May 2016, but is currently delayed due to severe civil and political violence related to the 2016 local elections, and is now hoped to complete in August. The research team are currently estimating whether a 15-20-month follow-up or a 20-26month follow-up is financially and practically possible, and if so, this would be expected to complete by December 2017. Since the submission of this manuscript, 16 countries in Southern, Eastern, Central and Northern Africa, South-East Asia and the Middle East have requested access to the programme in order to adapt it and take it to scale in their contexts (Additional file 1).

\section{Additional file}

Additional file 1: SPIRIT 2013 checklist: recommended items to address in a clinical trial protocol. (DOC $122 \mathrm{~KB}$ )

\begin{abstract}
Abbreviations
CASI, computer-assisted self-interview; ICAST, ISPCAN Child Abuse Screening Tools; ISPCAN, International Society for the Prevention of Child Abuse and Neglect; LMIC, low- and middle-income country; NGO, non-governmental organisation; PEPFAR, President's Emergency Plan for AIDS Relief; PLH, Parenting for Lifelong Health; RCT, randomised controlled trial; SAHA, Social and Health Assessment; UNICEF, United Nations Children's Emergency Fund; USAID, US Agency for International Development; WHO, World Health Organization
\end{abstract}

\section{Acknowledgements}

The team wishes to acknowledge resources made available from the Adolescent Well-being Research Programme, funded primarily by the Department for International Development, the Economic and Social Research Council's Impact Acceleration Account at the University of Oxford, the Eastern Cape Provincial Department of Social Development and the South African National Arts Council. In the broader PLH suite, we are grateful to our many donor partners. Please see http://www.who.int/violence_injury_prevention/violence/child/plh/en/for further details of both the full suite of programmes and our donor partners. The authors would also like to thank those who contributed to the study: Lorraine Sherr,

Tshiamo Petersen, Sibongile Tsoanyane, Christopher Mikton and Lulu Ncobo.

\section{Funding}

The study and intervention are supported by the European Research Council (ERC) under the European Union's Seventh Framework Programme (FP7) 2007-2013) with ERC grant agreement 313421, UNICEF Innocenti Office of Research, UNICEF South Africa, The John Fell Fund and the Leverhulme Trust (PLP-2014-095).

\section{Availability of data and materials}

Sinovuyo Teen manuals and programme materials will be made freely available online, and UNICEF has sponsored free printed versions. All research materials (i.e. questionnaires, study process materials and qualitative toolkit) will be made freely available on UNICEF and WHO websites. The study data will be made available on open-access websites such as the South African Data Archive and the European Clinical Trials database.

\section{Authors' contributions}

LC conceived of the study and drafted the manuscript for publication. FM participated in the design of the study and drafted an extensive study protocol. YS participated in the design of the study, drafted an extensive study protocol and performed sample size calculations. CLW provided overall guidance in RCT design and contributed to the development of the protocol. RHR participated in the design of the study and drafted an extensive study protocol. AR participated in the design of the study, drafted an extensive study protocol and trialled methods in the planning stages. CL participated in the design of the study and planned the statistical analyses. $\mathrm{JS}$ and DB participated in the design and trial of measures and helped draft the protocol. RC, CW, NS and PM participated in the design and adaptation of the trial through trial methods and community liaison in the planning stages. $J$ participated in the design of this study and provided feedback during protocol development. FG provided overall guidance in RCT design. $J D$ and $H L$ participated in the design of the qualitative study. MN and SDS developed innovative methods for use of audio-CASI with non-literate populations. ML participated in the design of the study and provided feedback on the manuscript. All authors read and approved the final manuscript. 


\section{Competing interests}

$J D, L C, J L, C L W$ were involved in the design of the Sinovuyo Teen programme. No profit or financial gain will be made from this programme.

\section{Ethics approval and consent to participate}

Ethical protocols were approved by the Faculty of Humanities Ethics Review Committee, University of Cape Town (PSY2014-001) and the Social Sciences and Humanities Inter-divisional Research Ethics Committee, University of Oxford (SSD/CUREC2/11-40), the European Research Council (ERC-2012-StG 313421-PACCASA) and South African provincial Departments of Social Development and Basic Education. Written voluntary informed consent is obtained from all participants and consent procedures are read aloud for those with limited literacy. Families do not receive monetary incentives, but receive 'thank-you packs' with a certificate of participation, a snack, stationery for school and toiletries at pre-test. At post-test, families receive a small food parcel. Confidentiality is maintained, except if participants are at risk of significant harm or request assistance. If participants report severe abuse, rape, recent suicide attempts or other significant harm, immediate referrals are made to child protection, health and HIV/AIDS services, with follow-up support.

\section{Author details}

${ }^{1}$ Centre for Evidence-Based Intervention, Department of Social Policy \& Intervention, University of Oxford, Barnett House, 32 Wellington Square, Oxford OX1 2ER, UK. ²Department of Psychiatry and Mental Health, University of Cape Town, Cape Town, South Africa. ${ }^{3}$ Institute of Criminology, University of Cambridge, , . . ${ }^{4}$ Department of Psychology, University of Cape Town, Cape Town, South Africa. ${ }^{5}$ Biostatistics Unit, South African Medical Research Council, Cape Town, South Africa. ${ }^{6}$ Clowns Without Borders South Africa, PO Box 18670, Durban 4014, South Africa. ${ }^{7}$ UNICEF, Offices of Research Innocenti, Florence, Italy.

\section{Received: 22 March 2016 Accepted: 28 June 2016}

\section{Published online: 19 July 2016}

\section{References}

1. Hillis S, Mercy J, Amobi A, Kress H. Global prevalence of past-year violence against children: a systematic review and minimum estimates. Pediatrics. 2016;137(3):1-13.

2. UNICEF. Hidden in plain sight: a statistical analysis of violence against children. New York: UNICEF; 2014.

3. Burton P, Ward CL, Artz L. The Optimus study on child abuse, violence and neglect in South Africa. Cape Town: The Centre for Justice and Crime Prevention; 2015.

4. Blakemore SJ. Imaging brain development: the adolescent brain. Neuroimage. 2012;61(2):397-406.

5. Mikton C, Butchart A. Child maltreatment prevention: a systematic review of reviews. Bull World Health Organ. 2009;87:353-61.

6. Lachman JM, Sherr L, Cluver L, Ward CL, Hutchings J, Gardner F. Integrating evidence with local contextual factors to develop a parenting program in low-income communities in South Africa. J Child Fam Stud. In press.

7. International Rescue Committee. Parents make the difference: findings from a randomised impact evaluation of a parenting program in rural Liberia. London: International Rescue Committee Report; 2014.

8. Annan J, Bunderviet T, Seban J, Costigan J. Urwaruka Rushasha. A randomised impact evaluation of village savings and loans associations and family-based interventions in Burundi. Washington, DC: International Rescue Committee and USAID; 2013.

9. Knerr W, Gardner F, Cluver L. Improving positive parenting skills and reducing harsh and abusive parenting in low- and middle-income countries: a systematic review. Prev Sci. 2013;14:352-363.

10. Barlow J, Johnston I, Kendrick D, Polnay L, Stewart-Brown S. Individual and group-based parenting programmes for the treatment of physical child abuse and neglect. Cochrane Database Syst Rev. 2006;3(Art. No: CD005463):1-20.

11. Gardner F, Montgomery P, Knerr W. Transporting evidence-based parenting programs for child problem behavior (age 3-10) between countries. Systematic review and meta-analysis. J Clin Child Adolesc Psychol. 2015;53:1-14

12. Eisner M, Humphreys DK, Wilson P, Gardner F. Disclosure of financial conflicts of interests in interventions to improve child psychosocial health: a crosssectional study. PLoS One. 2015;10(11):e0142803.

13. Cluver L, Lachman J, Ward C, Gardner F, T P, Hutchings J, Mikton C, Meinck F, Tsoanyane S. Development of a parenting support programme to prevent abuse of adolescents in South Africa: findings from a pilot pre-post study. Res Social Work Prac. In press.

14. Cluver L, Meinck F, Yakubovich A, Doubt J, Redfern A, Ward C, Salah N, De Stone S, Petersen T, Mpimpilashe $\mathrm{P}$, et al. Reducing child abuse amongst adolescents in low- and middle-income countries: a pre-post trial in South Africa. BMC Public Health. In review. in press.

15. Department of Health. Province of the Eastern Cape Annual Report 2010/11. Pretoria: Department of Health; 2012

16. Barlow J, Coren E, Stewart-Brown S. Meta-analysis of the effectiveness of parenting programmes in improving maternal psychosocial health. $\mathrm{Br} J \mathrm{Gen}$ Pract. 2002:52:223-33.

17. Gardner F, Burton J, Klimes I. Randomised controlled trial of a parenting intervention in the voluntary sector for reducing child conduct problems: outcomes and mechanisms of change. J Child Psychol Psychiatry. 2006:47(11):1123-32

18. Potterton J, Stewart A, Cooper P, Becker P. The effect of a basic home stimulation programme on the development of young children infected with HIV. Dev Med Child Neurol. 2010;52(6):547-51.

19. Higgins J, Green S (eds.). Cochrane Handbook for systematic reviews of interventions, Version 5.1.0. London: The Cochrane Collaboration; 2011.

20. Zolotor AJ, Runyan DK, Dunne MP, Jain D, Peturs HR, Ramirez C, Volkova E, Deb S, Lidchi V, Muhammad T et al. ISPCAN Child Abuse Screening Tool Children's Version (ICAST-C): instrument development and multi-national pilot testing. Child Abuse Negl. 2009;33(11):833-41.

21. Runyan DK, Dunne MP, Zolotor AJ, Madrid B, Jain D, Gerbaka B, Menick DM, Andreva-Miller I, Kasim MS, Choo WY et al. The development and piloting of the ISPCAN Child Abuse Screening Tool - Parent version (ICAST-P). Child Abuse Negl. 2009;33(11):826-32.

22. Frick P. The Alabama Parenting Questionnaire. Alabama: University of Alabama; 1991.

23. Achenbach T. Manual for the youth self-report and 1991 profile. Burlington: University of Vermont; 1991.

24. Berry J, Jones $\mathbf{W}$. The parental stress scale: initial psychometric evidence. J Soc Pers Relat. 1995;12:463-72.

25. Radloff $L$. The CES-D scale: a self-report depression scale for research in the general population. Appl Psychol Meas. 1977;1:385-401.

26. Kovacs M. Children's depression inventory. Niagara Falls: Multi-Health Systems; 1992.

27. Sheehan D, Shytle D, Milo K. MINI KID: mini international neuropsychiatric interview for children and adolescents, English version 4.0. Paris: University of South Florida, Tampa and Hopital de la Salpetriere; 2004.

28. Sherbourne C, Stewart A. The Medical Outcomes Survey (MOS) social support survey. Soc Sci Med. 1991;32:705-14.

29. Lown J. Development and validation of a financial self-efficacy scale. J Financ Couns Plann. 2011;22(1).p.54-63. http://ssrn.com/abstract=2001554.

30. Karlan D, Linden L. Loose knots: strong versus weak commitments to save for education in Uganda. Kampala: National Bureau of Economic Research; 2014.

31. Kast F, Meier S, Pomeranz D. Under-savers anonymous: evidence on selfhelp groups and peer pressure as a savings commitment device (Working Paper No. 18417). Kampala: National Bureau of Economic Research; 2011.

32. Hutchinson M. The Parent-Teen Sexual Risk Communication Scale (PTSRC-III). Nurs Res. 2007;56(1):1-8.

33. McCarty C, Doyle S. Parent-child communication (child/parent) (Fast Track Project Technical Report). Durham, NC: Duke University Press. 2001. http:// www.fasttrackproject.org/.

34. Ruchkin V, Schwab-Stone M, Vermeiren R. Social and Health Assessment (SAHA) psychometric development summary. New Haven: Yale University; 2004.

35. Ward C, Martin E, Theron C, Distiller G. Factors affecting resilience in children exposed to violence. S Afr J Psychol. 2007:37(1):165-87.

36. Adolescent Pathways Project. Social support scale: psychometric development summary. New York: New York University; 1992

37. Banerjee A, Duflo E. The economic lives of the poor. J Econ Perspect. 2007; 21(1):141-68.

38. Pulerwitz J, Barker R. Measuring attitudes toward gender norms among young men in Brazil: development and psychometric evaluation of the GEM Scale. Men Masculinities. 2008:10:322-38.

39. Lopman B, Barnabas R, Boerma T, Chawira J, Gaitskell K, Harrop T, Mason P, Donnelly C, Garnett G, Nyamukapa C Huskisson J, Dannhauser A, Vorster $\mathrm{HH}$, Nesmvuni AE et al. Creating and validating an algorithm to measure AIDS mortality in the adult population using verbal autopsy. Public Libr Sci Med. 2006;3(8):1273-81. 
40. Labadarios D, Steyn NP, Maunder E, MacIntryre U, Gericke G, Swart R, Huskisson J, Dannhauser A, Vorster HH, Nesmvuni AE et al. The National Food Consumption Survey (NFCS): South Africa, 1999. Public Health Nutr. 2005;8(5):533-43.

41. Pillay U, Roberts B, Rule S, editors. South African social attitudes: changing times, diverse voices. Cape Town: HSRC Press; 2006.

42. Dunne M, Zolotorb A, Runyanb D, Andreva-Millerc I, Yuen Chood W, Dunnea S, Dunnea S, Gerbakae B, Isaevaf O, Jaing D, Sham Kasimh M et al. ISPCAN Child Abuse Screening Tools Retrospective version (ICAST-R): Delphi study and field testing in seven countries. Child Abuse Negl. 2009;33(11):815-25.

43. Straus M, Hamby S, Boney-McCoy S, Sugarman D. The revised conflict tactics scales (CTS2): development and preliminary psychometric data. J Fam Issues. 1996;17:283-316.

44. Pulerwitz J, Gortmaker S, DeJong W. Measuring sexual relationship power in HIV/STD research. Sex Roles. 2000:42(7/8):637-60.

45. Gardner F, Connell A, Trentacosta CJ, Shaw DS, Dishion TJ, Wilson MN. Moderators of outcome in a brief family-centered intervention for preventing early problem behavior. J Consult Clin Psychol. 2009;77(3):543-53.

46. Gardner F, Hutchings J, Bywater T, Whitaker C. Who benefits and how does it work? Moderators and mediators of outcome in an effectiveness trial of a parenting intervention. J Clin Child Adolesc Psychol. 2010;39(4):568-80.

47. Hayes A. Introduction to mediation, moderation and conditional process analysis. New York: Guilford Press; 2012.

48. Raudenbush SW, Liu X. Statistical power and optimal design for multisite randomized trials. Psychol Methods. 2000;5(2):199-213.

49. Chen M, Chan KL. Effects of parenting programs on child maltreatment prevention: a meta-analysis. Trauma Violence Abuse. 2016;17(1):88-104.

\section{Submit your next manuscript to BioMed Central and we will help you at every step:}

- We accept pre-submission inquiries

- Our selector tool helps you to find the most relevant journal

- We provide round the clock customer support

- Convenient online submission

- Thorough peer review

- Inclusion in PubMed and all major indexing services

- Maximum visibility for your research

Submit your manuscript at www.biomedcentral.com/submit 